in endometrial cancer. In order to classify endometrial cancer more precisely, especially those with nonspecific molecular profile (NSMP), we aimed to determine the prognostic significance of 3 additional proteins in the institution's endometrial cancer cohort: $\beta$-catenin, L1 cell-adhesion molecule (L1CAM), and programmed death-ligand 1(PD-L1).

Methods: Endometrial cancer patients, surgically treated between 2014 and 2018 at our institution, underwent ProMisE classification using immunohistochemical (IHC) staining for p53 and 4 mismatch repair (MMR) proteins (MLH1, PMS2, MSH2, and MSH6) and hot spot sequencing of DNA polymerase epsilon (POLE) exonuclease domain via droplet digital polymerase chain reaction. Expression of $\beta$-catenin, L1CAM, and PD-L1 were studied by IHC staining. Then, we investigated the relationship between protein expression and progression-free survival (PFS).

Results: In total, 156 patients were included in this analysis: 136 (87.2\%) and 107 (68.6\%) had an endometrioid histologic type and early-stage disease, respectively. ProMisE classification assigned 45 (28.8\%), 16 (10.3\%), 70 (44.9\%), and 25 (16.0\%) to MMRd, POLEmut, NSMP (p53wt), and p53abn, respectively. Using 5\% of nuclear staining as a cut-off, we observed no difference in PFS between positive and negative $\beta$-catenin expression groups $(\mathrm{p}=0.465)$. Expression of PD-L1 was most frequent in the MMRd subgroup (48.9\%). Multivariate analysis adjusting confounders revealed that $\mathrm{PD}-\mathrm{L} 1 \geq 1 \%$ of tumor cells was identified as a favorable prognostic biomarker for PFS (adjusted hazard ratio $[\mathrm{HR}]=0.394$; $95 \%$ confidence interval $[\mathrm{CI}]=0.182-0.854 ; \mathrm{p}=0.018)$. However, PD-L1 expression was not predictive of prognosis among the NSMP subgroup. Using $10 \%$ of staining as a cut-off, high expression of L1CAM was most frequent in the p53abn subgroup (32.0\%). High expression of L1CAM was identified as a poor prognostic biomarker for PFS (adjusted HR=3.486; 95\% CI=1.577-7.707; $\mathrm{p}=0.002)$. L1CAM expression was predictive of prognosis among the NSMP subgroup. In subgroup analyses in endometrioid tumors, consistent results were observed.

Conclusion: While $\beta$-catenin was not informative for risk stratification, expression of PD-L1 and L1CAM were associated with disease recurrence. Especially, L1CAM expression further stratifies the risk of disease recurrence among the NSMP subgroup.

Poster (E09)

Endometrial Hyperplasia, Endometrial Intra-epithelial Neoplasia, and Endometrial Cancer

https://doi.org/10.3802/jgo.2021.32.S1.E09

\section{Interim analysis of immune-related endpoints of the mismatch repair deficient and proficient endometrial cancer (EC) cohorts from the garnet study}

Yoshiaki Kawano,,$^{1, *}$ Ana Oaknin, ${ }^{2}$ Lucy Gilbert, ${ }^{3}$ Anna Tinker, ${ }^{4}$ Renaud Sabatier, ${ }^{5}$ Valentina Boni ${ }^{6}$

'GlaxoSmithKline, Brentford, UK (yoshiaki.x.kawano@gsk.com)

${ }^{2}$ Vall d'Hebron Institute of Oncology, Barcelona, Spain

${ }^{3}$ McGill University, Montreal, Canada

${ }^{4} \mathrm{BC}$ Cancer, Vancouver, Canada

${ }^{5}$ Institut Paoli Calmettes, Marseille, France

${ }^{6}$ Hospital Universitario HM Sanchinarro, Madrid, Spain

Objective: GARNET (NCT02715284) is assessing the antitumor activity and safety of dostarlimab, an anti-programmed death-1 receptor therapy, in patients with solid tumors. We report investigator-assessed (IA) efficacy endpoints for endometrial cancer (EC) patients.

Methods: A phase 1, multicenter, open-label, single-arm, dose-escalation and cohort-expansion study. In 2 independent expansion cohorts of patients with recurrent/advanced EC (deficient mismatch repair [dMMR] and mismatch repair proficient [MMRp] determined by immunohistochemistry) that progressed on/after a platinum regimen, patients received dostarlimab $500 \mathrm{mg}$ IV Q3W for 4 cycles, then 1,000 mg Q6W until disease progression, discontinuation, or withdrawal. Primary endpoints of objective response rate (ORR), disease control rate (DCR), and duration of response (DOR) using RECIST v1.1 were reported previously (Oaknin ESMO 2020 LBA36). Immune-related (ir) ORR, irDCR, and irDOR by irRECIST, based on IA, were secondary endpoints.

Results: One hundred twenty-six dMMR and 145 MMRp patients were enrolled and dosed; 110 and 144 patients, respectively, had measurable disease at baseline. After median (range) follow-up of 16.5 (0.03-30.6) and 13.7 (0.03-33.1) months for dMMR and MMRp patients, irORR was $45.5 \%$ and $13.9 \%$, irDCR was $63.6 \%$ and $42.4 \%$, and irDOR was not reached and $12.2 \%$, respectively.

Conclusion: Efficacy endpoints by RECIST v1.1 and irRECIST were similar. irDCR was particularly interesting in MMRp patients, a group with a poorer prognosis, but the potential benefit awaits confirmation in ongoing randomized controlled studies.

Funding: GSK 213346; editorial support from Fishawack Health, funded by GSK.

This abstract was previously presented at the Society of Gynecologic Oncology 2021 Annual Meeting and is submitted on behalf of the original authors with their permission.

Poster (E10)

Endometrial Hyperplasia, Endometrial Intra-epithelial Neoplasia, and Endometrial Cancer

https://doi.org/10.3802/jgo.2021.32.S1.E10 